\title{
A Simple DNA Preparation Method for High Quality Polymerase Chain Reaction in Rice
}

\author{
Sung-Ryul Kim², Jungil Yang ${ }^{2}$, Gynheung $\mathrm{An}^{2}$, Kshirod K. Jena ${ }^{1}$ * \\ ${ }^{1}$ Plant Breeding, Genetics, and Biotechnology Division, International Rice Research Institute, Metro Manila 1226, Philippines \\ ${ }^{2}$ Crop Biotech Institute and Graduate School of Biotechnology, Kyung Hee University, Yongin 17104, Korea
}

\begin{abstract}
Preparation of DNA is cumbersome especially in the case of large numbers of plant samples. Several simple plant DNA preparation methods have been developed for use in conjunction with polymerase chain reaction (PCR) analysis. However, those methods have not been adopted widely for rice molecular analysis. We present a new, simple, and inexpensive method using tris-phosphate (TPE) ethylenediaminetetraacetic acid (EDTA) buffer (100 mM tris-HCl pH9.5, $1 \mathrm{M} \mathrm{KCl,} 10 \mathrm{mM}$ EDTA pH 8.0) without phenol-chloroform extraction and DNA precipitation steps. The method consists of five steps: leaf tissue grinding, incubating in TPE buffer at $65^{\circ} \mathrm{C}$ for 20 to 90 minutes, diluting extracts with water, centrifuging to sediment tissue debris, and transferring the supernatant for direct use in PCR or storage. Agarose gel analysis of the crude extracts indicated that the method produced intact genomic DNA (gDNA) from young and old leaves of both young seedlings and mature plants. Leaf sample size $(0.5$ to $8.0 \mathrm{~cm}$ long) for DNA preparation was less sensitive to PCR than the previous methods. DNA quality was tested through PCR amplification of various GC content regions and product sizes, and we obtained bands from all samples, indicating that the method produced suitable DNA quality for PCR. gDNAs were stable for longer than eight months at $4^{\circ} \mathrm{C}$. This protocol enabled one person to handle several hundred samples in a day and was tested through various PCR-gel analyses such as genotyping of rice T-DNA mutant lines, positional cloning of rice mutant, and high throughput marker-assisted breeding using allele-specific SNP/Indel markers.
\end{abstract}

Keywords DNA extraction, TPE, CTAB, PCR, Rice

\section{INTRODUCTION}

Polymerase chain reaction (PCR) has been highly utilized for various purposes in rice such as quantitative trait locus (QTL) analysis, positional cloning, marker-assisted breeding, and analysis of transgenic plants. Preparation of genomic DNA (gDNA) is cumbersome requirement for a high quality PCR procedure. Several methods from plant tissues have been developed for PCR analysis, but are not accepted widely in rice. It may come from several reasons such as the requirement of a DNA precipitation step using isopropyl alcohol (Dellaporta et al. 1983; Edwards et al. 1991; Monna et al. 2002; Hayashi et al. 2004), sensitivity to conditions and treatments (Saini et al. 1999; Kasajima et al. 2004), low PCR success rate $(<95 \%)$ from the extracted DNAs (Xin et al. 2003), and poor information about DNA stability and PCR efficiency (Wang et al. 1993). Eventually these methods might not significantly reduce time and labor and might also lead to poor reliability and reproducibility in PCR. As a result, the cetyltrimethylammonium bromide (CTAB) method is still predominantly used in rice (Yan et al. 2009; Fujita et al. 2013; Luo et al. 2013; Xiao et al. 2015) despite being a time and labor intensive method.

The handling of large number of samples for PCR prompted us to develop a simple DNA preparation method. Here, we described a new, simple, and inexpensive DNA preparation protocol for rice through modification and systematization of the previously reported tris-phosphate (TPE) buffer method (Thomson and Henry 1995). The suitability of the resulting DNA for PCR was intensively tested in this study.

Received January 4, 2016; Revised January 31, 2016; Accepted February 4, 2016; Published February 28, 2016

*Corresponding author Kshirod K. Jena, k.jena@irri.org, Tel: +63-2-580-5600, Fax: +63-2-891-1236 


\section{MATERIALS AND METHODS}

\section{Plant materials}

Leaf tissues were collected from seven indica varieties of Oryza sativa (NSIC Rc222, PSB Rc82, IR24, IR56, IR64, Irga427, and Parao), three japonica varieties of $O$. sativa (Nipponbare, Osmancik-97, and Taichung 65), the wild rice species $O$. rufipogon (Accession no. 106424), two intermediate breeding lines (YPF14-582 and YPF14-853), and a T-DNA mutant line (PFG_3A-62268). The T-DNA mutant line was grown at Kyung Hee University, South Korea and the other materials were grown in a screen house or paddy field at the International Rice Research Institute (IRRI), Philippines. Fresh leaves or leaves stored at $-20^{\circ} \mathrm{C}$ for several months (lines YPF14-582, YPF14-853, and PFG_3A-62268) were used for DNA preparation using our modified TPE method and the CTAB method (Kim et al. 2011).

\section{PCR amplification}

PCR amplification was conducted in $20 \mu$ of PCR mixture containing $1.5 \mu \mathrm{l}$ of rice leaf extract, $10 \mathrm{mM}$ Tris- $\mathrm{HCl}$ (pH 8.3), $1.5 \mathrm{mM} \mathrm{MgCl}, 50 \mathrm{mM} \mathrm{KCl}, 200 \mu \mathrm{M}$ of each dNTP, $0.25 \mu \mathrm{M}$ of each primer, and 1 unit of Taq DNA polymerase (SolGent, Daejeon, Korea; www.solgent. com/eng/). For amplification of the GC rich region with Chr07A primer set, $3 \mu$ l of BandDoctor (SolGent) was included in the reactions. The thermal cycling conditions were initial denaturation at $94^{\circ} \mathrm{C}$ for 3 minutes, 35 cycles of 25 seconds at $95^{\circ} \mathrm{C}, 25$ seconds at $55^{\circ} \mathrm{C}$, and 30 to 90 seconds at $72^{\circ} \mathrm{C}$, and final extension at $72^{\circ} \mathrm{C}$ for 5 minutes. The PCR primers used in this study are shown in Table 1. PCR product size and GC content were calculated based on Os-Nipponbare-Reference-IRGSP-1.0 sequence. For genotyping of rice T-DNA mutant line, we followed the procedure reported by Yi and An (2013) was followed.

\section{Protocol}

1. Prepare TPE buffer (100 mM Tris- $\mathrm{HCl} \mathrm{pH} 9.5,1 \mathrm{M}$ $\mathrm{KCl}, 10 \mathrm{mM}$ ethylenediaminetetraacetic acid [EDTA] $\mathrm{pH}$ 8.0). For example, for $100 \mathrm{ml}$ of TPE buffer, prepare $70 \mathrm{ml}$ of water, then add $10 \mathrm{ml}$ of $1 \mathrm{M}$ Tris- $\mathrm{HCl} \mathrm{pH} \mathrm{9.5,} 7.45 \mathrm{~g}$ of $\mathrm{KCl}$, and $2 \mathrm{ml}$ of $0.5 \mathrm{M}$ EDTA pH 8.0, and finally make it $100 \mathrm{ml}$ with water. Buffer can be stored at room temperature for 2 weeks.

2. Collect leaf tissue ( 2 to $4 \mathrm{~cm}$ long) in $2 \mathrm{ml}$ tube. Fresh leaf can be used immediately or stored at $-20^{\circ} \mathrm{C}$ for several months.

3. Grind leaf tissue. The leaf tissue with a metallic ball in $2 \mathrm{ml}$ tube will be frozen by dipping in liquid nitrogen and will be ground by high frequency strokes of a high throughput homogenizer such as TissueLyser II (Qiagen, Hilden, Germany; www. qiagen.com/) or Geno/Grinder (SPEX SamplePrep, Metuchen, NJ, USA; www.spexsampleprep.com/). Tissue should be broken well to help releasing gDNA from cells.

4. Add $200 \mu \mathrm{l}$ of TPE buffer immediately into each sample tube and mix well by vigorous hand shaking.

5. Incubate the samples at $65^{\circ} \mathrm{C}$ for 20 to 90 minutes.

Table 1. List of polymerase chain reaction primers used in this study.

\begin{tabular}{cll}
\hline \hline Primers & \multicolumn{1}{c}{ Forward primer $\left(5^{\prime} \rightarrow 3^{\prime}\right)$} & \multicolumn{1}{c}{ Reverse primer $\left(5^{\prime} \rightarrow 3^{\prime}\right)$} \\
\hline Chr02A & TTGACAACCACTCCTGTCCT & CCCTTCAACATGGTTGAGGT \\
Chr02B & TGGGCATGTTTCTGGAGACA & CTCGAATGGCTTCCAATGAC \\
Chr03A & GCTCCAAGAACTAATACAAGC & CTAGCTTGAGGGTTCCTGCA \\
Chr03B & TTGGCTTGATTTCCTGTGCTA & GCTTTCTGCTCCTGCTGTAA \\
Chr03C & TGCACCATAACTACACTTGCT & CTTGAATGCTTGCTGGTCGA \\
Chr06A & TTCCCATCTGCACTACCATAATCC & GAGCAGAGATGTGCTTTGCTACC \\
Chr06B & GAGATCAGTACTTGTACTAGC & TCAACTTACTCCCTCAGTCT \\
Chr07A & GACCTCACCTGCTATAGCTA & GCTCGATCGAGCCGATCAT \\
Chr08A & GGTTGTTCAGTGGCAATGTC & GCAAAAGTGCAGCTAACCAC \\
Chr09A & GCAAGTGCTCACCCAAGTG & AGCAACCACTGAGACAGCAT \\
\hline
\end{tabular}

\footnotetext{
${ }^{Z}$ The number in the primer name represents rice chromosome number.
} 
6. Add $1 \mathrm{ml}$ of water into each tube for dilution of extract, and then shake the tubes vigorously to mix.

7. Centrifuge the sample tubes at $13,000 \times g$ for 10 minutes at room temperature.

8. Take out the sample tubes carefully from the centrifuge and transfer as much supernatant as you need without any tissue debris to a new 1.5 - $\mathrm{ml}$ microfuge tube or a 96-well plate. Use $1.5 \mu 1$ of extract per 15 to $25 \mu \mathrm{l}$ of PCR mixture. Extracts can be stored at $4^{\circ} \mathrm{C}$ for at least eight months.

\section{RESULTS}

\section{Integrity of rice gDNA extracted by TPE method}

The integrity of gDNA was checked from two indica cultivars and two japonica cultivars using gel electrophoresis of the leaf extracts containing gDNA prepared by the TPE method. To examine the effect of each component of the TPE buffer, leaf extracts were also prepared using water and solutions in which one of the components was eliminated (PE, TE, and TP) from TPE buffer (Fig. 1A). No DNA or RNA bands were observed from the extracts prepared with water only, indicating that the water only may not be effective in extracting the nucleic acids or it may not protect the nucleic acids that are extracted from subsequent degradation. In the PE extracts, most RNAs were removed and also some of the gDNA was damaged. The extracts using the TE solution showed mainly RNA with faint intact gDNA bands and some degraded gDNA. Both TP and TPE solutions showed high-yielding intact gDNA with some RNAs. For longer stability of gDNA we

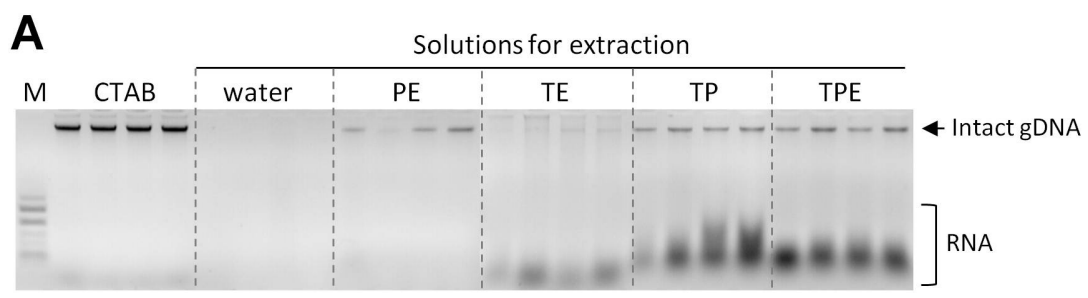

B
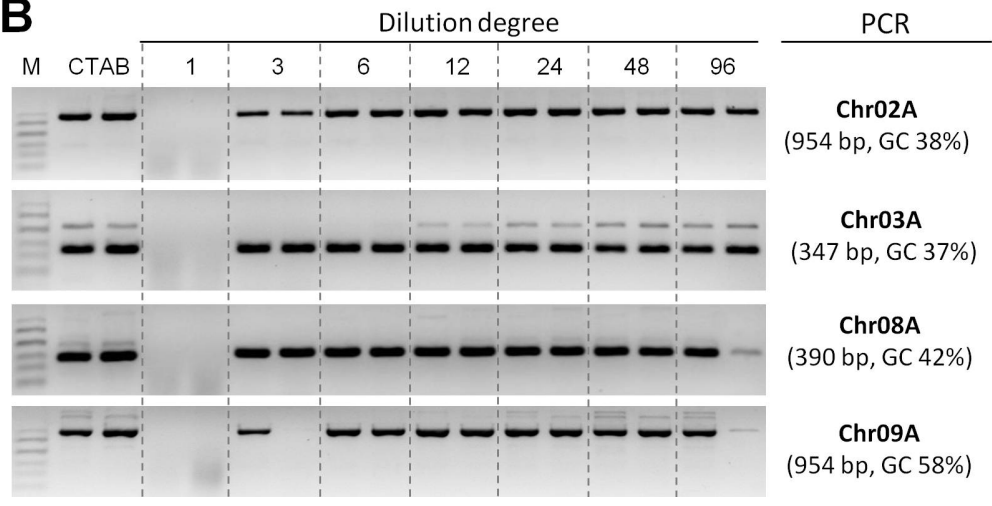

Fig. 1. Agarose gel image of leaf extracts and screening of the optimal dilution degree of the crude leaf extracts for polymerase chain reaction (PCR). (A) Gel image of leaf extracts. Leaf tissue (4 cm long) was collected from 15 to 80 days old plants of varieties NSIC Rc222 (lane 1), PSB Rc82 (lane 2), Nipponbare (lane 3), and Osmancik-97 (lane 4), respectively. Following extraction, the supernatants $(15 \mu \mathrm{l})$ were electrophoresed in $1 \%$ agarose gel. (B) PCR amplification efficiency according to dilution degree of leaf extracts. Leaf tissue $(2 \mathrm{~cm}$ long $)$ was prepared from 50 days-old plant of NSIC Rc222 (lane 1) and Taichung 65 (lane 2) as described. PCRs were performed with four primer sets. Product sizes and GC contents are shown next to the gel images. CTAB-extracted DNA was used as a control.

M: DNA size marker, CTAB: cetyltrimethylammonium bromide; $500 \mathrm{ng}$ of genomic DNA (gDNA) extracted by CTAB method, T: $100 \mathrm{mM}$ tris- $\mathrm{HCl} \mathrm{pH}$ 9.5, P: $1 \mathrm{M} \mathrm{KCl}$, E: $10 \mathrm{mM}$ ethylenediaminetetraacetic acid (EDTA) $\mathrm{pH} 8.0$. 
selected the TPE buffer.

\section{Optimization of dilution degree of the original leaf extracts}

In order to address the possible presence of inhibitors from the rice tissue and the high EDTA concentration in TPE buffer, the original extracts from two rice cultivars were diluted with double-distilled water up to 96 times and the optimal dilution degree was determined by PCR (Fig. 1B). Undiluted extracts failed to amplify PCR products in both cultivars with all four primer sets. No or poor amplifications were observed in three times dilution for Chr02A and Chr09A primers and in 96 times dilution for Chr08A and Chr09A primers. Six to 48 times dilution showed similar amplification to PCR of DNA extracted using the CTAB-method. We selected six times dilution because of the capacity of the microfuge tubes used $(2 \mathrm{ml})$.

\section{The influences of sample quantity and plant developmental stages on PCR efficiency}

Sample quantity and plant developmental stages can influence in DNA quantity and quality in DNA extraction. Leaf tissue segments of $0.5,1,2,4$, and $8 \mathrm{~cm}$ in length were sampled from the seedling (IR24 and NSIC Rc222) and the grain filling (Taichung 65 and IR64) stages of development. DNAs were prepared using the TPE method and subjected to PCR with four different primers (Fig. 2). In all conditions, PCR products of expected size were obtained regardless of sample sizes and developmental stages. However, small sample sizes $(0.5$ and $1 \mathrm{~cm}$ long) showed low efficiency in amplifying GC-rich (Fig. 2C) and larger (Fig. 2D) regions. When we used longer than 2-cm leaf

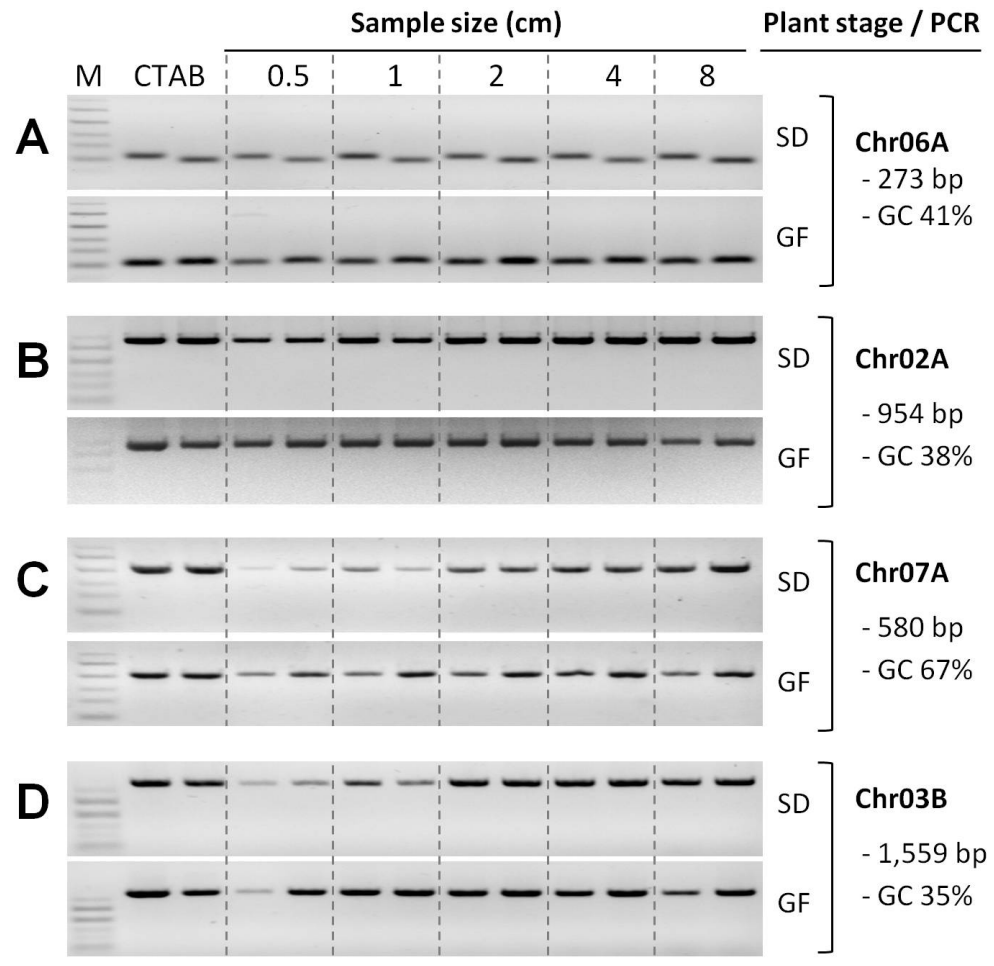

Fig. 2. The influences of sample quantity and plant developmental stages on polymerase chain reaction (PCR) of tris-phosphate (TPE)-extracted DNA. Leaf tissue $(0.5,1,2,4$, and $8 \mathrm{~cm}$ long) was collected from the seedling stage (SD, 20 days after germination) of IR24 and NSIC Rc222 and from the grain filling stage (GF, 10 days after pollination) of Taichung 65 and IR64, respectively. DNAs were prepared by TPE buffer with six times dilution. PCR efficiency was tested using Chr06A (A), Chr02A (B), Chr07A (C), and Chr03B (D) primers. Cetyltrimethylammonium bromide (CTAB)-extracted DNA was used as control.

M: DNA size marker. 
piece, PCR band intensity was similar to that of CTABmethod DNAs. These results indicate that TPE method is less sensitive to sample sizes and plant stages.

\section{Suitability of TPE-extracted DNA for PCR of GC-rich regions}

The efficiency of PCR amplification is highly influenced by the sequence context and product size of the target region together with DNA quality. It is relatively difficult to amplify GC rich region or large size PCR products. The suitability of DNAs extracted using the TPE method for PCR of difficult regions was evaluated using primers for the amplification of five different loci of high GC content (67\%) ranging from 1,250 to 1,987 bp in length (Fig. 3). Template DNAs were prepared from 10 rice cultivars and one wild species, O. rufipogon. PCR products of the expected size for the five different loci were amplified from all the samples, indicating that this protocol produced suitable DNA quality for PCR in rice.
The stability of DNAs extracted by the TPE method

gDNA stability is also an important factor when selecting DNA preparation methods for various downstream applications. To test DNA stability, the TPE-leaf extracts were stored at $4^{\circ} \mathrm{C}$ for 8 months, and then they were analyzed by agarose-gel electrophoresis and PCR amplification. The amount of high molecular weight gDNA from the stored extracts was the same as freshly extracted samples (Fig. 4A) and PCR amplification with Chr09A primer set showed the expected band sizes (Fig. 4B). This result indicated that gDNAs in the TPE extracts were stable at $4^{\circ} \mathrm{C}$ for at least 8 months.

\section{Applications of the simple DNA preparation method}

This protocol has been used for several PCR analyses such as genotyping of rice T-DNA mutant lines, transgene detection from transgenic rice plants, positional cloning of a rice mutant, selection of true F1 plants using rice microsatellite (RM) markers, and marker-assisted breeding using allele specific SNP/Indel markers (personal communi-

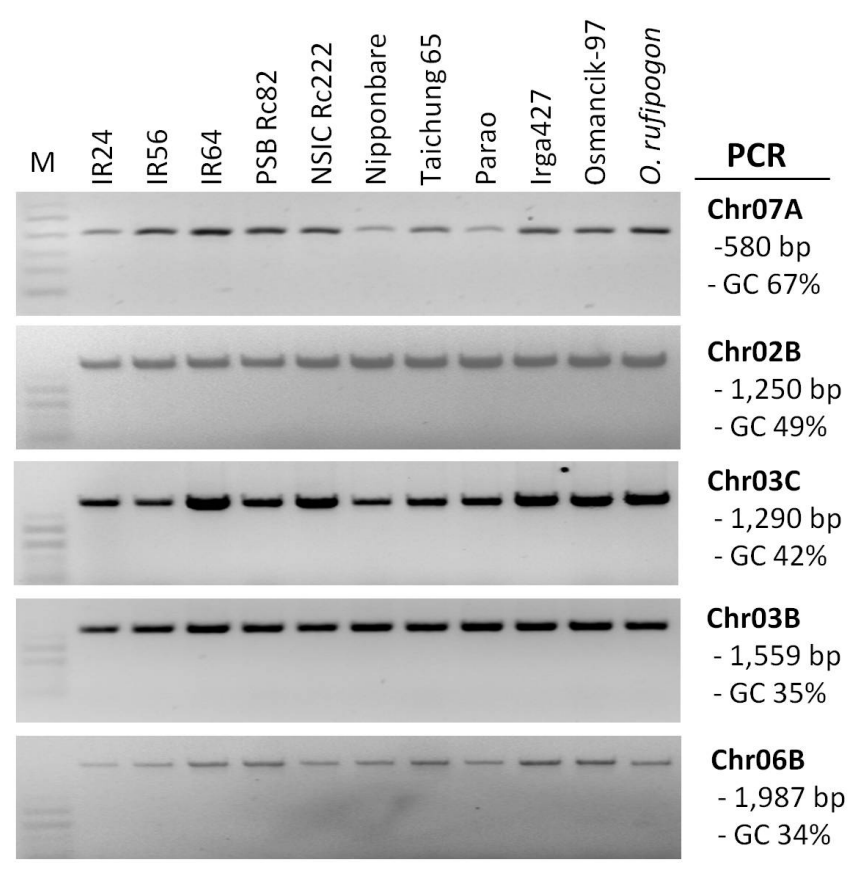

Fig. 3. DNA quality test by polymerase chain reaction (PCR) amplifications of regions of various GC content ( $34 \%$ to $67 \%$ ) and product sizes (580 to $1,987 \mathrm{bp}$ ). The leaf extracts were prepared based on the tris-phosphate protocol from 11 rice accessions. PCRs were performed with five primer sets.

M: DNA size marker. 


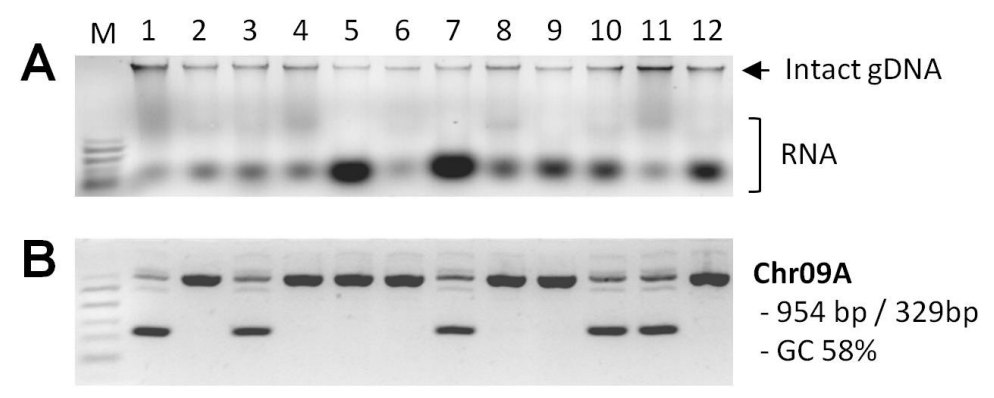

Fig. 4. DNA stability test. Leaf extracts were prepared by the tris-phosphate protocol from $12 \mathrm{BC}_{2} \mathrm{~F}_{2}$ plants of breeding line YPF14-853, and then they were stored at $4^{\circ} \mathrm{C}$ for 8 months. Genomic DNA integrity was tested by gel electrophoresis of $15 \mu \mathrm{l}$ of the stored extracts (A) and by polymerase chain reaction amplification of the stored extracts with Chr09A primer set (B).

M: DNA size marker.

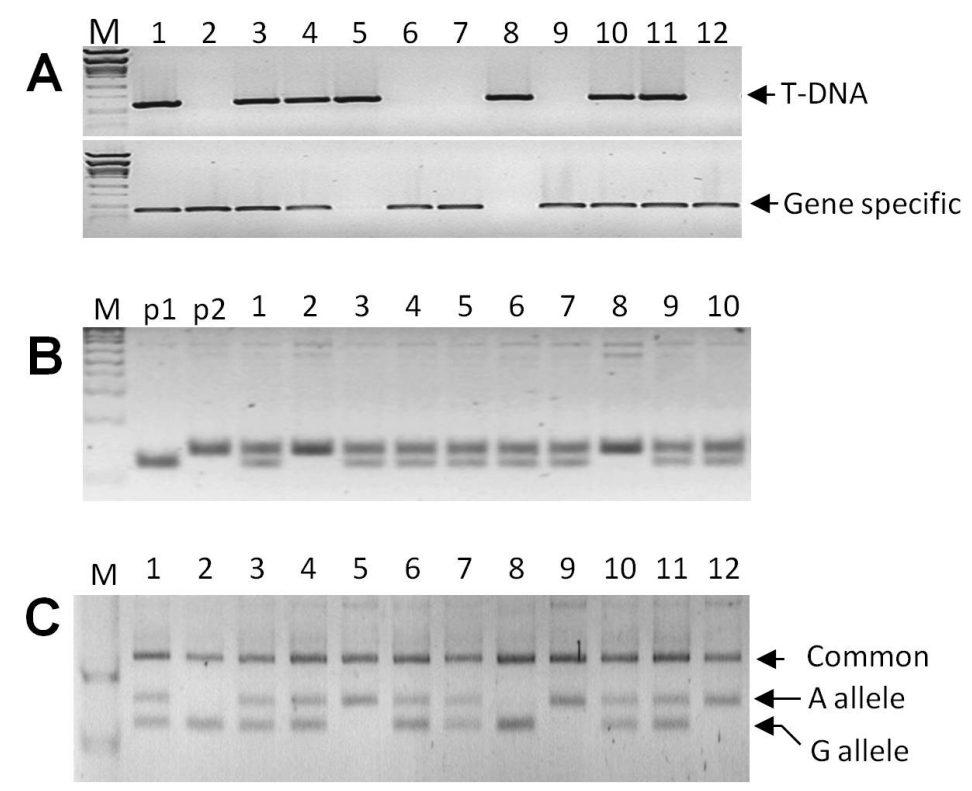

Fig. 5. Various polymerase chain reaction (PCR) applications using genomic DNA extracted by the TPE method. (A) Genotyping of $12 \mathrm{~T}_{2}$ plants of the rice T-DNA insertional mutant line, PFG_3A-52268. (B) $\mathrm{F}_{1}$ hybridity test using rice microsatellite $(\mathrm{RM})$ marker, RM 11 from $10 \mathrm{~F}_{1}$ plants. (C) Discrimination of SNP type DNA polymorphism for marker-assisted breeding. The A/G SNP located in the promoter region of Gnla gene which regulates grain number per panicle (Ashikari et al. 2005) was determined from $12 \mathrm{BC}_{1} \mathrm{~F}_{2}$ plants of the intermediate breeding line YPF14-582 using the tetra-primer PCR method (Ye et al. 2001).

M: DNA size marker, p1 and p2: parents of $F_{1}$.

cation with Prof. Laura Bartley, Univ. of Oklahoma, USA and Dr. Ramkumar Gandhimani, IRRI, Philippines). All tests were successful. As examples, genotyping of rice T-DNA mutant line, selection of true $\mathrm{F}_{1}$ plants using RM marker, and PCR-gel based SNP genotyping for markerassisted breeding are shown in Fig. 5 (Ye et al. 2001;
Ashikari et al. 2005). This simple DNA preparation protocol can be used for PCR-gel analysis for various purposes in rice. 


\section{DISCUSSION}

We described the simple DNA preparation method for PCR analysis in rice without hazardous organic chemicals (e.g., chloroform/phenol) or DNA precipitation. Its simplicity reduced time, labor, cost, and human error. The method produced stable intact gDNA from small piece of rice leaf and samples from various rice cultivars and transgenic plants produced uniform PCR efficiency.

The TPE buffer was originally developed by Thomson and Henry (1995) to develop a single-step protocol for preparation of plants DNA for PCRs. Small pieces of plant leaf tissue or embryos of cereal crops containing $20 \mu \mathrm{l}$ of TPE solution were incubated at $95^{\circ} \mathrm{C}$ for 10 minutes, and then $1 \mu$ of supernatant was used for PCR. The method was tested roughly in more than 10 plant species by PCR with three primer sets. Here we modified and systematized the previous protocol and we tested DNA integrity, DNA stability, and DNA quality for PCRs. Finally it was validated through intensive PCR applications. For development of a new protocol, we considered two major points: 1) the method should produce reliable DNA quality for PCR; and 2) the method should be significantly easier than the current popular method in rice, the CTAB method (Chen and Ronald 1999). To obtain homogeneous and consistent PCR results, we added tissue grinding and centrifugation steps from the original TPE protocol. These additional steps are likely to have improved the efficiency of the extraction and reduced the amount of contamination from possible inhibitors, thus resulting in uniform PCR band intensity among the samples. Though two steps were added, the total procedure was much simpler than the CTAB method, which requires chloroform extraction and DNA precipitation steps. Incubation at $65^{\circ} \mathrm{C}$ rather than $95^{\circ} \mathrm{C}$ is easier to achieve in the laboratory and was sufficient to obtain high gDNA yields from ground rice leaf tissues. The component analysis of TPE buffer showed that $1 \mathrm{M} \mathrm{KCl}$ was very important to obtain both high yield gDNA and intact gDNA (Fig. 1A). The presence of $10 \mathrm{mM}$ EDTA in extraction buffer might protect gDNA from endonuclease by immediate adding TPE buffer after tissue grinding. And the following heat incubation step $\left(65^{\circ} \mathrm{C}, 20\right.$ to 90 minutes) might denature many enzymes in leaf tissue including DNA degrading enzymes. These may lead to the long stability of gDNA at $4^{\circ} \mathrm{C}$. Regarding extract dilution, we usually used six times dilution. The dilution degree can be adjusted for better PCR efficiency because plant metabolites which inhibit PCR can be variable among rice accessions. Our protocol was less sensitive to plant stages, sample quantity, and incubation time than other simple and CTAB methods. These are advantages for obtaining uniform band intensity among samples and also reliable PCR results among batches. Actually the leaf extract contains gDNA as well as many other substances such as protein, starch, and metabolites and its color is green because of chlorophylls. But there were no significant difficulties to amplify GC rich regions and large products (Fig. 3). We have used this protocol for preparation of gDNA from more than 20,000 rice plants derived from the cross between the donor lines of rice yield-enhancing genes and 12 indica elite varieties as a recipient, and we obtained successful PCR genotyping results with allele-specific SNP/Indel markers. These results support that the simple protocol produces suitable and reproducible DNA quality for PCR genotyping in marker-assisted breeding programs. Moreover this protocol can be used for the preparation of gDNA for various PCR-gel analyses in rice.

\section{ACKNOWLEDGEMENTS}

We are grateful to the Global Rice Science Partnership (GRiSP) program (Grant No.: DRPC 2011-134) of IRRI for financial support. We thank the editorial team of IRRI Communications for English editing of the manuscript.

\section{REFERENCES}

Ashikari M, Sakakibara H, Lin S, Yamamoto T, Takashi T, Nishimura A, et al. 2005. Cytokinin oxidase regulates rice grain production. Science 309: 741-745.

Chen DH, Ronald PC. 1999. A rapid DNA minipreparation method suitable for AFLP and other PCR applications. Plant Mol. Biol. Rep. 17: 53-57.

Dellaporta SL, Wood J, Hicks JB. 1983. A plant DNA mini- 
preparation: Version II. Plant Mol. Biol. Rep. 1: 19-21.

Edwards K, Johnstone C, Thompson C. 1991. A simple and rapid method for the preparation of plant genomic DNA for PCR analysis. Nucleic Acids Res. 19: 1349.

Fujita D, Trijatmiko KR, Tagle AG, Sapasap MV, Koide Y, Sasaki K, et al. 2013. NAL1 allele from a rice landrace greatly increases yield in modern indica cultivars. Proc. Natl. Acad. Sci. U.S.A. 110: 20431-20436.

Hayashi K, Hashimoto N, Daigen M, Ashikawa I. 2004. Development of PCR-based SNP markers for rice blast resistance genes at the Piz locus. 2004. Theor. Appl. Genet. 108: 1212-1220.

Kasajima I, Ide Y, Ohkama-Ohtsu N, Hayashi H, Yoneyama T, Fujiwara T. 2004. A protocol for rapid DNA extraction from Arabidopsis thaliana for PCR analysis. Plant Mol. Biol. Rep. 22: 49-52.

Kim SR, Jeon JS, An G. 2011. Development of an efficient inverse PCR method for isolating gene tags from T-DNA insertional mutants in rice. Methods Mol. Biol. 678: 139-146.

Luo X, Ji SD, Yuan PR, Lee HS, Kim DM, Balkunde S, et al. 2013. QTL mapping reveals a tight linkage between QTLs for grain weight and panicle spikelet number in rice. Rice 6: 33.

Monna L, Kitazawa N, Yoshino R, Suzuki J, Masuda H, Maehara Y, et al. 2002. Positional cloning of rice semidwarfing gene, sd-1: rice "green revolution gene" encodes a mutant enzyme involved in gibberellin synthesis. DNA Res. 9: 11-17.

Saini HS, Shepherd M, Henry RJ. 1999. Microwave extraction of total genomic DNA from barley grains for use in PCR. J. Inst. Brew. 105: 185-190.

Thomson D, Henry R. 1995. Single-step protocol for preparation of plant tissue for analysis by PCR. Biotechniques 19: 394-397, 400.

Wang H, Qi M, Cutler AJ. 1993. A simple method of preparing plant samples for PCR. Nucleic Acids Res. 21: 4153-4154.

Xiao N, Huang WN, Li AH, Gao Y, Li YH, Pan CH, et al. 2015. Fine mapping of the qLOP2 and qPSR2-1 loci associated with chilling stress tolerance of wild rice seedlings. Theor. Appl. Genet. 128: 173-185.

Xin Z, Velten JP, Oliver MJ, Burke JJ. 2003. High-throughput DNA extraction method suitable for PCR. Biotechniques 34: 820-824, 826.

Yan CJ, Yan S, Yang YC, Zeng XH, Fang YW, Zeng SY, et al. 2009. Development of gene-tagged markers for quantitative trait loci underlying rice yield components. Euphytica 169: 215-226.

Ye S, Dhillon S, Ke X, Collins AR, Day IN. 2001. An efficient procedure for genotyping single nucleotide polymorphisms. Nucleic Acids Res. 29: E88.

Yi J, An G. 2013. Utilization of T-DNA tagging lines in rice. J. Plant Biol. 56: 85-90. 